\title{
Taxas de perda gestacional até 60 dias são afetadas por características cíclicas da égua receptora de embrião Mangalarga Marchador
}

\author{
[Rates of pregnancy loss up to 60 days are affected by cyclic characteristics of the \\ Mangalarga Marchador recipient mare] \\ N.C. Eulálio ${ }^{1}$, L.M. Borges ${ }^{1}$, E.P. Lopes $^{1}$, P.S. Gomes ${ }^{1}$, G.R. Valle ${ }^{2^{*}}$ \\ ${ }^{1}$ Médico veterinário autônomo \\ ${ }^{2}$ Departamento de Medicina Veterinária - PUC Minas em Betim, MG
}

\begin{abstract}
RESUMO
Foram avaliadas taxas de gestação aos 15 dias e perda gestacional entre 15 e 60 dias em 430 transferências de embrião (TE) em éguas Mangalarga Marchador. Diagnósticos de gestação foram realizados entre 15 e 60 dias após TE. Para avaliar os efeitos da duração da fase folicular da receptora, foram formados três grupos: até três dias ( $\leq 3 \mathrm{~d})$; quatro a seis dias $(4-6 \mathrm{~d})$; sete ou mais dias $(\geq 7 \mathrm{~d})$. Para avaliar os efeitos do tamanho do folículo pré-ovulatório da receptora, foram formados outros três grupos: menor ou igual a $35 \mathrm{~mm}(\varnothing \leq 35)$; maior que 35 e menor ou igual a $45 \mathrm{~mm}(35<\emptyset \leq 45)$; maior que $45 \mathrm{~mm}$ $(\varnothing>45)$. Os grupos foram comparados pelo teste qui-quadrado $(\mathrm{P}<0,05)$. Quanto à duração da fase folicular, as taxas de gestação foram semelhantes ( $\leq 3 \mathrm{~d}-83,1 \% ; 4-6 \mathrm{~d}-86,4 \% ; \geq 7 \mathrm{~d}-86,0 \%)$, e a perda gestacional maior em $\geq 7 \mathrm{~d}(24,4 \%)$ que em $\leq 3 \mathrm{~d}(12,0 \%)$ e $4-6 \mathrm{~d}(13,3 \%)$, estas semelhantes entre si. Quanto ao tamanho folicular, as taxas de gestação foram semelhantes $(\varnothing \leq 35-86,4 \% ; 35<\emptyset \leq 45-86,5 \%$; $\emptyset>45-81,9 \%)$, assim como as de perda gestacional $(\varnothing \leq 35-13,2 \% ; 35<\emptyset \leq 45-18,1 \% ; \varnothing>45-10,5 \%)$. Razões para a maior perda gestacional no grupo $\geq 7 \mathrm{~d}$ não foram esclarecidas, mas conclui-se que a duração da fase folicular pode ser fator de escolha de receptoras.
\end{abstract}

Palavras-chave: equino, transferência de embrião, receptora, características cíclicas, perda gestacional

\begin{abstract}
Pregnancy rates were evaluated at 15 days and pregnancy loss between 15 and 60 days on 430 embryo transfers (ET) in Mangalarga Marchador mares. Pregnancy diagnosis was performed between 15 and 60 days after ET. To evaluate the effects of the duration of the follicular phase of the recipient mare, three groups were formed: up to 3 days ( $\leq 3 d)$; 4 to 6 days (4-6d); 7 or more days ( $\geq 7 d)$. To evaluate the effects of the size of the pre-ovulatory follicle of the recipient mare, three other groups were formed: below or

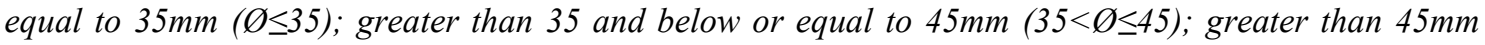
$(\varnothing>45)$. The groups were compared by Chi-square test $(P<0.05)$. Regarding the duration of the follicular phase, pregnancy rates were similar ( $\leq 3 d-83.1 \%$; $4-6 d-86.4 \%$; $\geq 7 d-86.0 \%)$, and greater pregnancy loss in $\geq 7 d(24.4 \%)$ than in $\leq 3 d(12.0 \%)$ and $4-6 d(13.3 \%)$, which were similar. Regarding the follicle

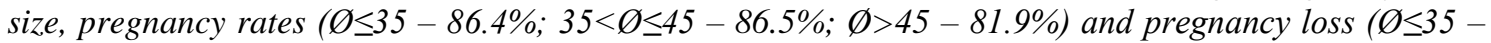
$13.2 \% ; 35<\varnothing \leq 45-18.1 \% ; \emptyset>45-10.5 \%)$ were similar. Reasons for the greatest pregnancy loss in the $\geq 7$ group have not been elucidated, but we conclude that the duration of the follicular phase may be a factor for choosing recipient mares.
\end{abstract}

Keywords: equine, embryo transfer, recipient mare, cyclical characteristics, pregnancy loss

Recebido em 2 de março de 2012

Aceito em 24 de janeiro de 2013

*Autor para correspondência (corresponding author)

E-mail: guilhermerv@pucminas.br 


\section{INTRODUÇÃO}

A transferência de embriões (TE) em equinos alcança hoje índices de fertilidade maiores que $75 \%$ aos 15 dias, graças à grande evolução da técnica desde sua primeira descrição nos anos 1970 por Ogury e Tsutsumi (McKinnon e Squires, 2007).

Diversos fatores ligados à técnica utilizada, aos embriões e a fatores intrínsecos e extrínsecos das receptoras são descritos como influenciadores das taxas de manutenção da gestação em receptoras de embrião (Squires et al., 1999; McKinnon e Squires, 2007), inclusive para a raça Mangalarga Marchador no Brasil (Lopes et al., 2011), mas não há menção na literatura consultada às características do estro da receptora como um desses fatores.

Classicamente as características ováricas e comportamentais durante o estro de éguas envolvem uma fase folicular que se inicia quando um folículo atinge diâmetro de 25 a $30 \mathrm{~mm}$, com duração média de $7,7 \pm 1,8$ dias, coincidente com a duração do estro comportamental. A velocidade média de crescimento do diâmetro folicular nessa fase, até a ovulação, é de 2,5 a $2,7 \mathrm{~mm} /$ dia, com redução dessa velocidade a partir de dois a quatro dias prévios à ovulação, esta ocorrendo de 24 a 48 horas antes do final do estro. A ovulação ocorre quando o folículo atinge, em média, 40 a $45 \mathrm{~mm}$ de diâmetro, usualmente a partir de $35 \mathrm{~mm}$, mas podendo ocorrer entre 20 e $60 \mathrm{~mm}$ (Pierson, 1993; Newcombe, 1996; Bergfeldt e Adams, 2007; Newcombe, 2007).

Em condições tropicais (Sudeste do Brasil), são encontrados dados semelhantes: período médio decorrido entre o início do estro e a ovulação de 7,2 dias; taxa de crescimento folicular de 2,5 a $2,6 \mathrm{~mm} /$ dia; tamanho médio dos folículos à

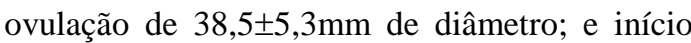
de manifestação comportamental de estro com folículo de $30 \pm 7 \mathrm{~mm}$ de diâmetro (Valle et al., 2005; Peres et al., 2006).

O período de duração da fase folicular e o tamanho do folículo à ovulação não parecem influenciar a fertilidade de éguas (Valle et al., 2005). No entanto, a influência dessas características do estro da receptora de embriões sobre a sua capacidade de manutenção de embriões transferidos não parece ter sido ainda avaliada. Diante disso, o objetivo deste trabalho foi verificar se a duração da fase folicular e o tamanho do folículo pré-ovulatório de receptoras de embrião equino afetam as taxas de sobrevivência embrionária até 60 dias de gestação.

\section{MATERIAL E MÉTODOS}

Foram avaliados dados reprodutivos de 430 TEs de 116 éguas doadoras da raça Mangalarga Marchador, realizadas em receptoras da mesma raça em Minas Gerais, região Sudeste do Brasil, durante três estações reprodutivas.

Doadoras e receptoras eram mantidas em pastagem de gramíneas com acesso livre a água e sal mineral, recebendo Penisetum purpureum picado e/ou feno de coast-cross como fonte suplementar de volumoso, além de concentrado comercial.

Doadoras e receptoras eram examinadas por palpação transretal e ultrassonografia a cada 48 horas até que fosse detectado um folículo com $30 \mathrm{~mm}$ de diâmetro em um dos ovários, passando a ser examinadas a cada 24 horas até a ovulação. As doadoras eram submetidas à monta natural ou inseminadas artificialmente a cada 48 horas a partir da detecção de folículo de $35 \mathrm{~mm}$ de diâmetro até a ovulação.

Os embriões foram coletados entre o oitavo e $10^{\circ}$ dias após a ovulação, por meio de lavagens uterinas com solução ringer com lactato aquecida a $35^{\circ} \mathrm{C}$ (Alvarenga et al., 1993) e transferidos por via transcervical, conforme técnica de rotina (Squires et al., 2003). Todas as TEs utilizaram embriões de qualidade grau 1 ou 2, numa escala de 1 a 5, em que 1 é o melhor (McKinnon e Squires, 1988). Os diagnósticos de gestação foram realizados por meio de ultrassonografia (Pycock, 2007) aos 15, 30, 45 e 60 dias após a ovulação da doadora.

As receptoras tinham a idade estimada pelo método de cronologia dentária (Silva et al., 2003) e foram previamente selecionadas quanto à higidez genital. No momento de cada TE, a receptora utilizada era escolhida, dentre as disponíveis, com base na proximidade de ovulação com a doadora e critérios de qualidade uterina avaliados por palpação transretal e 
ultrassonografia, que incluíam boa tonicidade cervical, boas tonicidade e tubularidade uterina e presença de edema endometrial com ausência de fluido luminal.

Foram considerados nesta análise apenas dados de TEs em que a receptora tenha tido ovulação simples e, além disso, em que não tenha ocorrido regressão do tamanho folicular a uma dimensão menor que $30 \mathrm{~mm}$ de diâmetro antes da ovulação.

Para avaliar os efeitos do período de duração da fase folicular da receptora (tempo decorrido entre a detecção de folículo de $30 \mathrm{~mm}$ de diâmetro até a ovulação) sobre as taxas de gestação aos 15 dias e taxas de perdas gestacionais entre 15 e 60 dias, os dados foram agrupados em: até três dias $(\leq 3 \mathrm{~d})$; quatro a seis dias (4-6d); e sete ou mais dias ( $\geq 7 \mathrm{~d})$. Para avaliar os efeitos do diâmetro do folículo da receptora à ovulação sobre as mesmas taxas, os dados foram reagrupados em: diâmetro menor ou igual a $35 \mathrm{~mm}(\varnothing \leq 35)$; diâmetro maior que 35 e menor ou igual a $45 \mathrm{~mm}(35<\varnothing \leq 45)$; e diâmetro maior que $45 \mathrm{~mm}(\varnothing>45)$.

Para cada avaliação, os grupos foram comparados entre si quanto às taxas de gestação aos 15 dias e às taxas de perda gestacional total (15 a 60 dias) e parcial (15 a 30; 30 a 45; e 45 a 60 dias) pelo teste qui-quadrado. As comparações entre grupos quanto a demais características reprodutivas, como idade do embrião, qualidade do embrião, sincronia doadora/receptora, idade da receptora, duração da fase folicular da receptora e tamanho do folículo pré-ovulatório da receptora, após avaliação de normalidade dos dados por meio do teste de Kolmogorov-Smirnov, foram realizadas utilizando-se o teste de Kruskal-Wallis. A existência de correlação entre a duração da fase folicular e o diâmetro do folículo pré-ovulatório das receptoras foi avaliada considerando-se o coeficiente de correlação de Spearman.

\section{RESULTADOS}

De maneira geral, a média de idade dos embriões transferidos foi de 8,2 $\pm 0,5$ dias; qualidade do embrião grau $1,1 \pm 0,5$; sincronismo doadora/receptora de $-2,7 \pm 1,8$ dias; idade das receptoras de 10,0 $\pm 4,8$ anos; duração da fase folicular de 5,0 $\pm 2,8$ dias; e tamanho do folículo pré-ovulatório de $42,8 \pm 6,4 \mathrm{~mm}$ de diâmetro. A taxa geral de gestação aos 15 dias foi de $85,4 \%$ (367/430); e a perda gestacional total dos 15 aos 60 dias de $15,3 \%$ (56/367), das quais $6,5 \%$ (24/367) entre 15 e 30 dias, 4,9\% (18/367) entre 30 e 45 dias, e $3,8 \%(14 / 367)$ entre 45 e 60 dias de gestação.

Para os grupos formados de acordo com a duração da fase folicular (Tab. 1), observou-se que o tamanho do folículo pré-ovulatório da receptora aumentou à medida que aumentou a duração da fase folicular da receptora $(\mathrm{P}<0,05)$, enquanto as demais características reprodutivas foram semelhantes entre grupos $(\mathrm{P}>0,05)$. As taxas de gestação aos 15 dias foram semelhantes entre grupos $(\mathrm{P}>0,05)$, entretanto a taxa de perda gestacional entre 15 e 60 dias foi maior $(\mathrm{P}<0,05)$ no grupo $\geq 7 \mathrm{~d}$ comparada à dos grupos $\leq 3 \mathrm{~d}$ e 4 $6 \mathrm{~d}$, nestes últimos semelhantes entre si $(\mathrm{P}>0,05)$. Avaliadas as taxas de perda gestacional por período, estas foram semelhantes entre grupos dos 15 aos 30 e dos 30 aos 45 dias ( $P>0,05)$, mas foi detectada maior perda dos 45 aos 60 dias no grupo $\geq 7 \mathrm{~d}$ comparada à do grupo $\leq 3 \mathrm{~d}(\mathrm{P}=0,05)$, com o grupo 4-6d em posição intermediária $(\mathrm{P}>0,05)$.

Para os grupos formados de acordo com o tamanho do folículo pré-ovulatório (Tab. 2), observou-se que a duração da fase folicular aumentou à medida que aumentou o tamanho do folículo pré-ovulatório da receptora $(\mathrm{P}<0,05)$, enquanto as demais características reprodutivas foram semelhantes entre grupos $(\mathrm{P}>0,05)$. As taxas de gestação aos 15 dias e as taxas de perda gestacional total e por período foram semelhantes entre grupos $(\mathrm{P}>0,05)$.

Avaliada a existência de correlação entre duração da fase folicular e tamanho do folículo préovulatório das receptoras, obteve-se correlação positiva e significativa $(\mathrm{R}=0,56 ; \mathrm{P}<0,0001)$.

\section{DISCUSSÃO}

A similaridade de idade e qualidade dos embriões transferidos, bem como de sincronia doadora/receptora e idade das receptoras entre os grupos (Tab. 1 e 2), revela que todas as TEs foram realizadas em condições semelhantes entre grupos experimentais. A sincronia média entre doadora e receptora foi de $-2,7 \pm 1,8$ dias, dentro do considerado ideal de +1 a -3 (Squires e Seidl, 1995), inclusive para a raça Mangalarga 
Marchador, identificada como de +1 a -4 (Fleury et al., 1989). Lopes et al. (2011) relatam que as melhores taxas de gestação foram obtidas com sincronia de -3 a -2 em 830 TEs realizadas na raça Mangalarga Marchador.

Tabela 1. Características reprodutivas de éguas Mangalarga Marchador receptoras de embrião de acordo com o período de duração da sua fase folicular (folículo de $30 \mathrm{~mm}$ de diâmetro até a ovulação)

\begin{tabular}{|c|c|c|c|}
\hline \multirow{2}{*}{ Característica reprodutiva } & \multicolumn{3}{|c|}{ Duração da fase folicular (dias) } \\
\hline & $\leq 3 \mathrm{~d}$ & $4-6 \mathrm{~d}$ & $\geq 7 \mathrm{~d}$ \\
\hline Número de TEs & 130 & 191 & 100 \\
\hline Fase folicular receptora (dias) & $2,6 \pm 0,7 \mathrm{~A}$ & $4,8 \pm 0,8 \mathrm{~B}$ & $8,8 \pm 2,9 \mathrm{C}$ \\
\hline Idade embrião (dias) & $8,2 \pm 0,5$ & $8,2 \pm 0,4$ & $8,2 \pm 0,4$ \\
\hline Qualidade embrião (1-5) ${ }^{1}$ & $1,2 \pm 0,4$ & $1,2 \pm 0,5$ & $1,1 \pm 0,3$ \\
\hline Sincronia doadora/receptora ${ }^{2}$ & $-2,6 \pm 1,7$ & $-2,7 \pm 1,7$ & $-2,7 \pm 2,0$ \\
\hline Idade receptora (dias) & $11,0 \pm 5,5$ & $10,4 \pm 4,6$ & $8,4 \pm 4,2$ \\
\hline Folículo pré-ov. receptora (mm) & $39,5 \pm 5,4 \mathrm{~A}$ & $42,6 \pm 5,7 \mathrm{~B}$ & $47,6 \pm 6,0 \mathrm{C}$ \\
\hline Taxa gestação aos 15 dias (\%) & $108 / 130(83,1)$ & $165 / 191(86,4)$ & $86 / 100(86,0)$ \\
\hline \multicolumn{4}{|l|}{ Perda gestacional $(\%)$} \\
\hline total (15 a 60 dias $)$ & $13 / 108(12,0) \mathrm{A}$ & $22 / 165(13,3) \mathrm{A}$ & $21 / 86(24,4) B$ \\
\hline 15 a 30 dias & $5 / 108(4,6)$ & $10 / 165(6,1)$ & $9 / 86(10,5)$ \\
\hline 30 a 45 dias & $6 / 103(5,8)$ & $6 / 155(3,9)$ & $6 / 77(7,8)$ \\
\hline 45 a 60 dias & $2 / 98(2,0) \mathrm{a}$ & $6 / 149(4,0) \mathrm{ab}$ & $6 / 71(8,5) b$ \\
\hline
\end{tabular}

1) Classificação segundo McKinnon e Squires (1988).

2) Valor negativo indica o número de dias em que a receptora ovulou após a doadora.

$\leq 3 \mathrm{~d}$, fase folicular de até 3 dias; 4-6d, fase folicular de 4 a 6 dias; $\geq 7 \mathrm{~d}$, fase folicular de 7 ou mais dias;

A, B e C indicam diferença significativa entre grupos $(\mathrm{P}<0,05)$.

$\mathrm{a}$ e $\mathrm{b}$ indicam diferença entre grupos $(\mathrm{P}=0,05)$.

Tabela 2. Características reprodutivas de éguas Mangalarga Marchador receptoras de embrião de acordo com o diâmetro $(\mathrm{mm})$ do seu folículo pré-ovulatório

\begin{tabular}{lccc}
\multirow{2}{*}{ Característica reprodutiva } & \multicolumn{3}{c}{ Diâmetro $(\varnothing)$ do folículo pré-ovulatório $(\mathrm{mm})^{3}$} \\
\cline { 2 - 4 } & $\varnothing \leq 35$ & $35<\varnothing \leq 45$ & $\emptyset>45$ \\
\hline Número de TEs & 88 & 237 & 105 \\
Folículo pré-ov. receptora (mm) & $33,9 \pm 2,2 \mathrm{~A}$ & $42,4 \pm 2,5 \mathrm{~B}$ & $51,4 \pm 2,9 \mathrm{C}$ \\
Idade embrião (dias) & $8,2 \pm 0,5$ & $8,2 \pm 0,4$ & $8,2 \pm 0,5$ \\
Qualidade embrião (1-5) & $1,2 \pm 0,5$ & $1,1 \pm 0,4$ & $1,2 \pm 0,5$ \\
Sincronia doadora/receptora & $-2,5 \pm 1,9$ & $-2,6 \pm 1,7$ & $-2,8 \pm 1,8$ \\
Idade receptora (dias) & $11,7 \pm 5,8$ & $9,6 \pm 4,7$ & $10,1 \pm 4,1$ \\
Fase folicular receptora (dias) & $3,7 \pm 1,9 \mathrm{~A}$ & $4,8 \pm 2,2 \mathrm{~B}$ & $6,8 \pm 3,5 \mathrm{C}$ \\
Taxa gestação aos 15 dias (\%) & $76 / 88(86,4)$ & $205 / 237(86,5)$ & $86 / 105(81,9)$ \\
Perda gestacional (\%) & & & \\
$\quad$ total (15 a 60 dias) & $10 / 76(13,2)$ & $37 / 205(18,1)$ & $9 / 86(10,5)$ \\
$\quad 15$ a 30 dias & $7 / 76(9,2)$ & $14 / 205(6,8)$ & $3 / 86(3,5)$ \\
30 a 45 dias & $2 / 74(2,7)$ & $12 / 191(6,3)$ & $4 / 83(4,8)$ \\
$\quad 45$ a 60 dias & $1 / 72(1,2)$ & $11 / 179(6,1)$ & $2 / 79(2,5)$ \\
\hline
\end{tabular}

1) Classificação segundo McKinnon e Squires (1988).

2) Valor negativo indica o número de dias em que a receptora ovulou após a doadora.

3) $\varnothing \leq 35$, diâmetro folicular menor ou igual a $35 \mathrm{~mm} ; 35<\varnothing \leq 45$, diâmetro folicular maior que 35 e menor ou igual a $45 \mathrm{~mm}$; $\varnothing>45$, diâmetro folicular maior que $45 \mathrm{~mm}$.

$\mathrm{A}, \mathrm{B}$ e C indicam diferença significativa entre grupos $(\mathrm{P}<0,05)$.

A taxa de concepção geral aos 15 dias de $85,4 \%$ foi maior que as de 65 a $75 \%$ (Squires et al., 1999), e com a raça Mangalarga Marchador de
73,4\% (Lopes et al., 2011) e 74,9\% (Fleury et al., 2001). Já a taxa geral de perda gestacional entre 15 e 60 dias de $15,3 \%$ pode ser considerada 
semelhante à de $16 \%$ entre 12 e 50 dias (Carnevale et al., 2000), mas maior que a de $11,7 \%$ (Taveiros et al., 2008) e a de $12,2 \%$ (Lopes et al., 2011), as duas últimas obtidas com a raça Mangalarga Marchador. Entretanto, esses dados confirmam as boas taxas de gestação aos 15 dias e taxas de perda gestacional até 60 dias obtidas em TEs nessa raça no Brasil. As perdas embrionárias em equinos variam de 2,5 a $25 \%$ (Vanderwall e Newcombe, 2007), e não parecem ser diferentes entre gestações em receptoras ou matrizes artificialmente inseminadas (Villahoz et al., 1985).

Observou-se uma correlação positiva altamente significativa $(\mathrm{R}=0,56 ; \quad \mathrm{P}<0,0001) \quad$ entre $\mathrm{O}$ diâmetro do folículo pré-ovulatório e a duração da fase folicular das receptoras, confirmando dados da literatura (Valle et al., 2005). Todavia, os efeitos da duração do estro e do tamanho do folículo pré-ovulatório sobre a fertilidade de éguas ainda não são claros na literatura. Esses fatores, quando relativos à égua receptora de embrião, não são capazes de exercer influência sobre a concepção e sobrevivência embrionária na primeira semana de vida do embrião, já que ele é gerado e mantido até o momento da TE (a partir do sétimo dia de vida) na doadora. Porém, não se sabe se tais fatores podem exercer algum efeito sobre as taxas de gestação após a TE, quando o embrião está na receptora.

Trum (1950) observou taxas de gestação menores quando os estros eram muito curtos (até dois dias) ou longos (10 ou mais dias), sendo, naquela época, atribuída aos estros longos menor fertilidade devido à maior contaminação uterina. Isso foi confirmado pelo uso de técnicas assépticas de inseminação artificial (Pickett et al., 1987) que levaram a resultados semelhantes de fertilidade entre estros longos e curtos (Valle et al., 2005), independentemente do número de inseminações por ciclo realizadas (Valle et al., 2000).

Para receptoras de embrião, os efeitos de fases foliculares longas sobre as taxas de gestação e as perdas gestacionais, entretanto, certamente seriam outros não relacionados às condições de cópula ou à inseminação artificial, pelas quais não são submetidas. As taxas de gestação semelhantes entre diferentes grupos de duração de fase folicular (Tab. 1) indicam que não houve efeito desse fator sobre a taxa de sobrevivência embrionária na primeira semana de vida na receptora (entre a TE e diagnóstico de gestação aos 15 dias). Entretanto, a partir desse momento, quando o embrião perde sua cápsula, fixa-se no endométrio e inicia um desenvolvimento embrionário mais intenso e placentação (Ginther, 1992), observou-se maior taxa de perda gestacional naquelas receptoras de fase folicular mais longa, especialmente entre 45 e 60 dias de gestação (Tab. 1). Nessa etapa da gestação, os efeitos advindos da fase folicular longa podem se tornar importantes, afetando a sobrevivência do concepto, o que não ocorreria em etapa anterior.

Em doadoras de ovócitos destinados à maturação in vitro, o tamanho do folículo puncionado é um importante aspecto relacionado à qualidade do ovócito e à eficiência na produção de embriões viáveis em diversas espécies (Mermillod et al., 2008), inclusive a equina (Goudet et al., 1997). No entanto, apesar de seu maior tamanho estar relacionado à maior fertilidade em novilhas (Perry et al., 2007), essa situação não é observada em éguas inseminadas (Valle et al., 2005) e não parece influenciar a taxa de gestação e sobrevivência embrionária em receptoras de embrião equino (Tab. 2).

Folículos com $25 \mathrm{~mm}$ de diâmetro ou mais produzem estrógeno suficiente para manter elevadas concentrações circulantes desse hormônio até a ovulação na égua (Pierson, 1993; Newcombe, 2007), coincidindo com o período de exposição dos órgãos genitais a concentrações elevadas de estrógeno circulante. $\mathrm{O}$ maior diâmetro de folículos antrais de éguas está relacionado à maior concentração de esteroides no líquido folicular (Baltsen et al., 2001), mas a produção folicular de esteroides no ovário equino mostrou ser dependente do grau de vascularização folicular, e não de seu diâmetro (YoungLai, 1971). Goudet et al. (1997) verificaram que a concentração de estrógenos no fluido de folículos pré-ovulatórios de éguas era muito variável, não sendo correlacionada com o diâmetro folicular. Em novilhas, entretanto, maior diâmetro folicular está relacionado com maiores concentrações de estrógenos circulantes (Perry et al., 2007).

A produção folicular de fatores que pudessem influenciar a habilidade de manutenção embrionária após 15 dias em receptoras equinas, como o estradiol-17 $\beta$ (Pierson, 1993; 
Newcombe, 2007), e sua manutenção por período longo, como nas fêmeas de fase folicular longa, poderiam ter causado, de alguma forma, maiores taxas de perda gestacional nessas receptoras. O papel do estrógeno produzido durante a fase folicular sobre o metabolismo uterino e a sobrevivência embrionária foi demonstrado em ovelhas (Miller et al., 1977), sendo a formação de receptores para progesterona no aparelho genital feminino estimulado pelo estrógeno em diversas espécies (Valle et al., 2007). Todavia, o uso de estradiol$17 \beta$ exógeno em éguas no anestro não interferiu na quantidade de receptores uterinos para progesterona (McDowell et al., 1999), e menor quantidade desses receptores foi identificada no epitélio endometrial de éguas nas fases iniciais da gestação em comparação com o estro (McDowell et al., 1999; Hartt et al., 2005). Porém, como o papel do estrógeno produzido durante o estro sobre a atividade uterina nas fases iniciais da gestação não está estabelecido para a égua, seu envolvimento nos resultados aqui obtidos é apenas uma hipótese que deve ser futuramente investigada.

\section{CONCLUSÃO}

Conclui-se que a duração da fase folicular da receptora deve ser um critério de escolha de receptoras no momento da transferência de embrião na espécie equina. Neste aspecto, receptoras com fase folicular maior que sete dias devem ser evitadas, por apresentarem maiores taxas de perda gestacional até 60 dias.

\section{AGRADECIMENTOS}

Este artigo contou com o apoio da FAPEMIG para publicação.

\section{REFERÊNCIAS}

ALVARENGA, M.A.; LANDIM, F.C.; MEIRA, C. Modifications in the technique used to recover equine embryos. Eq. Vet. J., Suppl., n.15, p.111$112,1993$.

BALTSEN, M.; BØGH, I.B.; BYSKOV, A.G. Content of meiosis activating sterols in equine follicular fluids: correlation to follicular size and dominance. Theriogenology, v.56, p.133-145, 2001.
BERGFELT, D.R.; ADAMS, G.P. Ovulation and corpus luteum development. In: SAMPER, J.C.; PYCOCK, J.F.; MCKINNON, A.O. (Ed) Current therapy in equine reproduction. Sant Louis: Saunders Elsevier, 2007. p.1-13.

CARNEVALE, E.M.; RAMIREZ, R.J.; SQUIRES, E.L. et al. Factors affecting pregnancy rates and early embryonic death after equine embryo transfer. Theriogenology, v.54, p.965-979, 2000.

FLEURY, J.J.; COSTA NETO, J.B.F.; ALVARENGA, M.A. Results from an embryo transfer programme with Mangalarga mares in Brazil. Eq. Vet. J., Suppl., n.8, p.73-74, 1989.

FLEURY, J.J.; PINTO, A.J.; MARQUES, A. et $a l$. Fatores que afetam a recuperação embrionária e os índices de prenhez após transferência transcervical em equinos da raça Mangalarga. Braz. J. Vet. Res. Anim. Sci., v.38, p.29-33, 2001.

GINTHER, O.J. Reproductive biology of the mare: basic and applied aspects. 2.ed, Madison: Equiservices, 1992. 642p.

GOUDET, G.; BEZARD, J.; DUCHAMP, G. et al. Equine oocyte competence for nuclear and cytoplasmic in vitro maturation: effect of follicle size and hormonal environment. Biol. Reprod., v.57, p.232-245, 1997.

HARTT, L.S.; CARLING, S.J.; JOYCE, M.M. et al. Temporal and spatial associations of oestrogen receptor alpha and progesterone receptor in the endometrium of cyclic and early pregnant mares. Reproduction, v.130, p.241-250, 2005.

LOPES, E.P.; SIQUEIRA, J.B.; PINHO, R.O. et al. Reproductive parameters of Mangalarga Marchador mares in a commercial embryo transfer programme. Reprod. Dom. Anim., v.46, p.261-267, 2011.

MCDOWELL, K.J.; ADAMS, M.H.; ADAM, C.Y. et al. Changes in equine endometrial oestrogen receptor alpha and progesterone receptor mRNAs during the oestrous cycle, early pregnancy and after treatment with exogenous steroids. J. Reprod. Fertil., v.117, p.135-142, 1999.

MCKINNON, A.O;; SQUIRES, E.L. Embryo transfer and related technologies. In: SAMPER, J.C.; PYCOCK, J.F.; MCKINNON, A.O. (Ed) Current therapy in equine reproduction. Sant Louis: Saunders Elsevier, 2007. p.319-334. 
MCKINNON, A.O.; SQUIRES, E.L. Morphological assessment of equine embryo. J. Am. Vet. Med. Assoc., v.192, p.401-406, 1988.

MERMILLOD, P.; DALBIÈS-TRAN, R.; UZBEKOVA, S. et al. Factors affecting oocyte quality: who is driving the follicle? Reprod. Dom. Anim., Suppl., n.43, p.393-400, 2008.

MILLER, B.G.; MOORE, N.W.; MURPHY, L. et $a l$. Early pregnancy in the ewe: effects of oestradiol and progesterone on uterine metabolism and on embryo survival. Aust. J. Biol. Sci., v.30, p.279288, 1977.

NEWCOMBE, J.R. The follicle: practical aspects of follicle control. In: SAMPER, J.C.; PYCOCK, J.F.; MCKINNON, A.O. (Ed) Current therapy in equine reproduction. Sant Louis: Saunders Elsevier, 2007. p.14-21.

NEWCOMBE, J.R. Ultrassonography of ovulation and development of the corpus luteum in the mare: a personal view. Eq. Vet. Educ., v.8, p.47-58, 1996.

PERES, K.R.; ALVARENGA, F.C.L.; ALVARENGA, M.A. Utilização do primeiro ciclo ovulatório da estação reprodutiva para produção de embriões em éguas sob condições tropicais. Braz. J. Vet. Res. Anim. Sci., v.43, p.270-279, 2006.

PERRY, G.A.; SMITH, M.F.; ROBERTS, A.J. et al. Relationship between size of the ovulatory follicle and pregnancy success in beef heifers. $J$. Anim. Sci., v.85, p.684-689, 2007.

PICKETT, B.W.; SQUIRES, E.L.; MCKINNON, A.O. Procedures for collection, evaluation and utilization of stallion semen for artificial insemination. Fort Collins: Colorado State University, Animal Reproduction Laboratory, 1987. $125 \mathrm{p}$.

PIERSON, R.A. Folliculogenesis and ovulation. In: MCKINNON, A.O.; VOSS, J.L. (Ed) Equine reproduction. Malvern: Lea \& Febiger, 1993. p.161-171.

PYCOCK, J.F. Pregnancy diagnosis in the mare. In: SAMPER, J.C.; PYCOCK, J.F.; MCKINNON, A.O. (Ed) Current therapy in equine reproduction. Sant Louis: Saunders Elsevier, 2007, p.335-342.

SILVA, M.F.; GOMES, T.; DIAS, A.S. et al. Estimativa da idade dos equinos através do exame dentário. Rev. Port. Cienc. Vet., v.98, p.103-110, 2003.
SQUIRES, E.L.; CARNEVALE, E.M.; MCCUE, P.M. et al. Embryo technologies in the horse. Theriogenology, v.59, p.151-170, 2003.

SQUIRES, E.L.; MCCUE, P.M.; VANDERWALL, D.K. The current status of equine embryo transfer. Theriogenology, v.51, p.91-104, 1999.

SQUIRES, E.L.; SEIDEL, G.E. Collection and transfer of equine embryos. Bulletin $n^{\circ} 8$. Fort Collins: Colorado State University, Animal Reproduction and Biotechnology Laboratory, 1995. p.24-26.

TAVEIROS, A.W.; MELO, P.R.M.; MACHADO, P.P. et al. Perda de concepto em programa de inseminação artificial e de transferência de embriões em equino da raça Mangalarga Marchador. Med. Vet. (Brasil), v.2, p.28-33, 2008.

TRUM, B.F. The estrous cycle of the mare. Cornell Vet., v.40, p.17-23, 1950.

VALLE, G.R.; CASSALI, G.D.; NOGUEIRA, J.C. et al. Nuclear estrogen and progesterone receptors in the oviduct of heifers under natural and superovulated estrous cycles. Anim. Reprod. Sci., v.101, p.28-37, 2007.

VALLE, G.R.; SILVA FILHO, J.M.; PALHARES, M.S. Características cíclicas e ovulatórias de éguas mestiças em Minas Gerais, Brasil. Arq. Cienc. Vet. Zool. UNIPAR, v.8, p.155-163, 2005.

VALLE, G.R.; SILVA FILHO, J.M.; PALHARES, M.S. et al. Efeito do número de inseminações artificiais sobre a fertilidade de éguas inseminadas com sêmen diluído, resfriado a $14^{\circ} \mathrm{C}$ e transportado. Rev. Bras. Zootec., v.29, p.1721-1726, 2000.

VANDERWALL, D.K.; NEWCOMBE, J.R. Early embryonic loss. In: SAMPER, J.C.; PYCOCK, J.F.; MCKINNON, A.O. (Ed) Current therapy in equine reproduction. Sant Louis: Saunders Elsevier, 2007, p.374-383.

VILLAHOZ, M.D.; SQUIRES, E.L.; VOSS, J.L. et al. Some observations on early embryonic death in mares. Theriogenology, v.23, p.915-924, 1985.

YOUNGLAI, E.V. Steroid content of the equine ovary during the reproductive cycle. J. Endocrinol., v.50, p.589-597, 1971. 